\title{
Ação educativa com adolescentes do Ensino Médio: Uma reflexão sobre
} autoimagem e transtornos alimentares

Educational action with High School adolescents: a reflection on self-image and food disorders

\section{Acción educativa con adolescentes de la Escuela Secundaria: una reflexión} sobre la imagen automática y los trastornos alimentarios

Recebido: 25/05/2020 | Revisado: 25/05/2020 | Aceito: 26/05/2020 | Publicado: 07/06/2020

Alicyregina Simião Silva

ORCID: https://orcid.org/0000-0002-8337-2728

Universidade da Integração Internacional da Lusofonia Afro - Brasileira, Brasil

E-mail: alicy.reginasilva@outlook.com

Janiel Ferreira Felício

ORCID: https://orcid.org/0000-0002-5601-0086

Universidade da Integração Internacional da Lusofonia Afro - Brasileira, Brasil

E-mail: janielferreirafelicio@gmail.com

Inara da Silva de Moura

ORCID: https://orcid.org/0000-0003-3612-0541

Universidade da Integração Internacional da Lusofonia Afro - Brasileira, Brasil

E-mail: inaramoura123@gmail.com

Luzia Camila Coêlho Ferreira

ORCID: https://orcid.org/0000-0003-0508-084X

Universidade da Integração Internacional da Lusofonia Afro - Brasileira, Brasil

E-mail: camila.coelho6400@gmail.com

José Carlos Gomes de Sousa

ORCID: https://orcid.org/0000-0002-3454-4650

Universidade da Integração Internacional da Lusofonia Afro - Brasileira, Brasil

E-mail: carlosgomesunilab@gmail.com

Carolina Maria de Lima Carvalho

ORCID: https://orcid.org/0000-0002-5173-5360

Universidade da Integração Internacional da Lusofonia Afro - Brasileira, Brasil 


\title{
Resumo
}

O modo como o indivíduo percebe o próprio corpo é modificado ao longo do tempo, e isso ocorre de modo mais intenso durante a adolescência. A insatisfação corporal é destacada como um dos importantes fatores considerados para o diagnóstico de transtornos alimentares sendo também um dos desencadeantes para a ocorrência destes. Dessa forma, o seguinte estudo tem como objetivo relatar uma experiência de educação em saúde realizada com adolescentes de uma Escola Profissional do Ensino Médio em um município do Maciço de Baturité, formação geológica localizada no sertão central cearense, abordando a temática da autoimagem corporal e suas implicações para a ocorrência de Transtornos Alimentares. A atividade ocorreu por meio de etapas que continham dinâmicas de interação e introdução da temática, bem como abordagem do conteúdo de forma dialogada. Diante do exposto, ainda há déficits em se abordar sobre a temática em ambiente escolar e em outros meios em que o adolescente está inserido. Portanto, o papel dos setores educacionais aliados aos de saúde são percebidos como áreas que devem estar articuladas de forma a garantir maior suporte e assistência aos jovens e seus familiares, caracterizando a atuação da equipe multiprofissional no aspecto da atenção psicossocial.

Palavras-chave: Autoimagem; Adolescente; Saúde mental; Promoção da saúde; Transtornos da alimentação.

\begin{abstract}
The way the individual perceives his own body is modified over time, and this occurs more intensely during adolescence. Body dissatisfaction is highlighted as one of the important factors considered for the diagnosis of eating disorders and is also one of the triggers for their occurrence. Thus, the following study aims to report a health education experience carried out with adolescents from a Professional High School in a municipality of Maciço de Baturite, geological formation located in the central hinterland of Ceará, addressing the theme of body self-image and its implications for the occurrence of Eating Disorders. The activity took place through stages that contained dynamics of interaction and introduction of the theme, as well as approaching the content in a dialogical way. Given the above, there are still deficits in addressing the theme in the school environment and in other environments in which the adolescent is inserted. Therefore, the role of educational sectors allied to health are perceived as areas that must be articulated in order to guarantee greater support and assistance to young
\end{abstract}


people and their families, characterizing the performance of the multiprofessional team in the aspect of psychosocial care.

Keywords: Self-image; Adolescent; Mental health; Promotion of health; Eating disorders.

\section{Resumen}

La forma en que el individuo percibe su propio cuerpo se modifica con el tiempo, y esto ocurre más intensamente durante la adolescencia. La insatisfacción corporal se destaca como uno de los factores importantes considerados para el diagnóstico de los trastornos alimentarios y también es uno de los factores desencadenantes de su aparición. Por lo tanto, el siguiente estudio tiene como objetivo informar una experiencia de educación para la salud realizada con adolescentes de una escuela secundaria profesional en un municipio de Maciço de Baturité, formación geológica ubicada en el interior central de Ceará, abordando el tema de la autoimagen corporal y su implicaciones para la aparición de trastornos alimentarios. La actividad se llevó a cabo a través de etapas que contenían dinámicas de interacción e introducción del tema, además de abordar el contenido de manera dialógica. En vista de lo anterior, todavía hay déficits para abordar el tema en el entorno escolar y en otros entornos en los que se inserta el adolescente. Por lo tanto, el papel de los sectores educativos aliados a la salud se percibe como áreas que deben articularse para garantizar un mayor apoyo y asistencia a los jóvenes y sus familias, caracterizando el desempeño del equipo multiprofesional en el aspecto de la atención psicosocial.

Palabras clave: Autoimagen; Adolescente; Salud mental; Promoción de la salud; Los trastornos alimentarios.

\section{Introdução}

O modo como o indivíduo percebe o próprio corpo é modificado ao longo do tempo, e isso ocorre de modo mais intenso durante a adolescência. Nesse contexto, é possível afirmar que a insatisfação relacionada à autoimagem é um problema vivenciado em diferentes localidades do mundo (Murari \& Dorneles, 2018). A exigência imposta pela mídia e pela sociedade na busca pelo corpo ideal exerce influência significativa sobre o modo como os sujeitos percebem a si mesmos diante do espelho. O desejo de seguir e alcançar os padrões de beleza atuais e, muitas vezes, a impossibilidade de transformá-los em realidade, produz insatisfação com a imagem corporal (Fontenele; Ramos; Goiabeira; Cutrim; Galvão \& Noronha, 2019). 
A Organização Mundial da Saúde (OMS) define a adolescência como uma fase da vida que se estende dos 10 aos 19 anos de idade. Nesse período, podem ocorrer diversas mudanças auxiliadas pela puberdade, e essas abrangem modificações físicas, psíquicas e sociais. As alterações corporais são notáveis, e podem ser grandemente influenciadas pela genética, pelos hábitos de vida e por padrões alimentares (Carvalho; Pereira \& Barros, 2018).

Diante de tantas alterações físicas e emocionais, a insatisfação com o corpo se torna uma queixa frequente entre adolescentes, o que aumenta a chance destes se tornarem insatisfeitos com a própria imagem, elevando o risco para a ocorrência e desenvolvimento de transtornos alimentares, como a bulimia e a anorexia. Fatores como distorção da imagem corporal e baixa autoestima reforçam uma busca demasiada pelo emagrecimento levando a atitudes como a realização exagerada de exercícios físicos, jejuns e o uso de laxantes e diuréticos de forma intensa visando a perda de peso (Murari \& Dorneles, 2018).

O processo da adolescência também é marcado por uma constante busca pela inserção social e adequação a determinados grupos com os quais o sujeito apresenta afinidade. A aparência física mostra-se, nesse sentido, como importante fator que contribui para o processo de aceitação e inserção social do adolescente, de modo que o corpo passa a representar o sucesso ou fracasso no contexto das relações afetivas, profissionais e sociais, determinando, de algum modo, a felicidade e os principais valores dos indivíduos (Ribeiro; Silva; Santos; Albuquerque \& Pichelli, 2015).

Vale ainda salientar que os meios de comunicação devem ser considerados não somente pela influência e contribuição na propagação de padrões de corpo ou de beleza ideais, como também pela disseminação de ações que visam alcançar esse modelo por meios muitas vezes perigosos e danosos a curto ou longo prazo. Pode-se destacar, nesse aspecto, o exemplo de dietas impróprias e insuficientes do ponto de vista nutricional ou a realização de atividades físicas excessivas sem o devido acompanhamento profissional, que são incentivadas e propagadas, em grande parte, através das redes sociais (Murari \& Dorneles, 2018).

De acordo com o Manual Diagnóstico e Estatístico de Transtornos Mentais (2014), os transtornos alimentares são caracterizados por alterações e perturbações persistentes na alimentação ou nos comportamentos relacionados à esta que alteram o consumo e a absorção dos alimentos, comprometendo de maneira significativa a saúde física e o funcionamento psicossocial dos indivíduos. São exemplos destes transtornos a anorexia e a bulimia nervosa, sendo considerados também os mais prevalentes em adolescentes. A anorexia nervosa possui como características principais a restrição na ingestão calórica alimentar acompanhada da 
perturbação com relação a percepção da forma corporal, além de comportamentos persistentes que interferem no ganho de peso causados pelo medo intenso de engordar. A bulimia nervosa é definida como um conjunto de ações que geram episódios recorrentes de compulsão alimentar e comportamentos inapropriados e compensatórios que visam impedir o ganho de peso. Nesse aspecto, a insatisfação corporal é destacada como um dos importantes fatores considerados para o diagnóstico de transtornos alimentares sendo também um dos desencadeantes para a ocorrência destes.

Os transtornos alimentares são responsáveis por causar um conjunto de danos e complicações físicas e psicológicas nos indivíduos acometidos. Os principais prejuízos físicos incluem alterações fisiológicas, déficit no crescimento e alterações hormonais. Os problemas psicossociais mais prevalentes são isolamento social e desenvolvimento de transtorno depressivo e de ansiedade, que elevam o risco de suicídio (Carvalho, 2019).

Segundo Teixeira et al. (2020), os sofrimentos psíquicos vivenciados na adolescência, possuem influência significativa na fase adulta. Durante este período, os sintomas relacionados à depressão, transtornos alimentares e de ansiedade apresentam-se de modo ainda mais intenso, e precisam ser percebidos precocemente, para a elaboração de cuidados que visam garantir um tratamento eficaz e a minimização dos danos físicos e psíquicos. A enfermagem apresenta-se como importante ferramenta no melhor manejo dos casos, de modo a orientar os diferentes públicos em ações que auxiliem no bem-estar físico e emocional, agregando assim, diferentes estratégias de promoção da saúde mental.

Pode-se destacar também a relevância de ações que visam a prevenção e promoção da saúde mental de adolescentes e jovens. Tais atividades podem ser realizadas em diferentes contextos, entre estes o ambiente escolar mostra-se como um dos espaços mais valorizados e importantes para esse público, visto que representa um importante meio de socialização e desenvolvimento, devendo também ser considerado como um rico campo para a realização de intervenções e práticas educativas de saúde (Grealish; Hunter; Emsley; Murrells \& Morrison 2017).

Dessa forma, o seguinte estudo tem como objetivo relatar uma experiência de educação em saúde realizada com adolescentes de uma Escola Profissional do Ensino Médio. A atividade buscou abordar a temática da autoimagem corporal e suas implicações para a ocorrência de Transtornos Alimentares, possibilitando o autocuidado e visando a prevenção e promoção da saúde física e mental do público envolvido. 


\section{Metodologia}

Trata-se de um estudo descritivo, do tipo relato de experiência de natureza qualitativa como preconizam Pereira et al. (2018). Nesse contexto, foi realizada, no dia 04 de junho de 2019, uma ação educativa organizada por cinco estudantes de enfermagem da Universidade da Integração Internacional da Lusofonia Afro-Brasileira. Os organizadores da ação cursavam, no momento, a disciplina Processo de Cuidar na Saúde Mental. A atividade ocorreu na Escola de Ensino e Educação Profissional Adolfo Ferreira de Souza, localizada na cidade de Redenção-CE. Os principais participantes da ação foram alunos do terceiro ano do Ensino Médio que pertenciam ao curso técnico de enfermagem. De modo que a atividade contou com a participação de 90 pessoas.

A ação educativa desenvolveu-se em etapas consecutivas e inter-relacionadas. Inicialmente foi realizada uma dinâmica quebra-gelo, intitulada como "Corredor do cuidado", visando promover maior interação entre os participantes da atividade. Em seguida se realizou a dinâmica "Colagem de depoimentos e notícias", tomando como estratégia analisar o conhecimento prévio dos participantes a respeito da temática, seguida da exposição do conteúdo por meio de palestra dialogada e recurso visual, abordando conceitos, sintomatologia, tratamento e prevenção, estes relacionados aos temas Distúrbios da autoimagem e distúrbios alimentares. Para a concretização da atividade realizou-se uma dinâmica final denominada "Dúvidas embaixo das carteiras", visando recapitular o conteúdo exposto e promover um momento de feedback da turma a respeito da ação realizada.

Por se tratar de um relato de experiência, e por não envolver a exposição dos participantes ou a coleta de dados, não foi necessário solicitar a aprovação do comitê de ética em pesquisa. Entretanto, foi solicitada a autorização da direção e coordenação da escola, através de requerimento formal da instituição proponente da intervenção. É importante também ressaltar que os alunos presentes na ação educativa consentiram, livremente, em participar da atividade.

\section{Resultados e Discussão}

Após a apresentação inicial da equipe organizadora e explicação sobre a finalidade e importância da atividade para os professores e alunos envolvidos, iniciou-se a realização das etapas que constituíram a ação educativa. Os momentos da ação foram divididos cronologicamente e estão devidamente descritos a seguir. 


\subsection{Primeira etapa: Dinâmica inicial "Corredor do cuidado"}

O primeiro momento da ação educativa consistiu na realização de uma atividade inicial denominada "Dinâmica do Corredor do Cuidado". Nesse momento, os alunos fizeram duas filas formando uma estrutura semelhante a um corredor, onde cada participante deveria passar e receber algum tipo de cuidado dos demais. Os cuidados incluíam elogios, um gesto de carinho, massagens e abraços. Esta etapa foi elaborada com intuito de possibilitar uma maior interação entre os participantes da atividade, e como forma de promover um momento de relaxamento, considerando os inúmeros aspectos estressores aos quais os estudantes estão expostos, incluindo as exigências escolares e familiares, consideradas fatores geradores de tensão que contribuem para tornar jovens e adolescentes mais vulneráveis a alterações e desequilíbrios psicológicos.

Segundo Santos (2017), a proximidade temporal com relação ao vestibular e o iminente ingresso no mercado de trabalho, associados a dificuldade na decisão com relação a carreira profissional, podem causar constantes episódios de estresse e ansiedade. Por esse motivo, a realização de ações e atividades que visem promover um momento de alívio e liberação da constante tensão ao qual o adolescente está exposto, apresenta-se como um importante instrumento de promoção da saúde mental e possível prevenção de futuras psicopatologias.

Durante a realização desse momento da atividade os participantes se mostraram bastante receptivos a dinâmica e foi possível notar o quanto esse momento de cuidado e afeto foi relevante para estes, de modo que a ação apresentou tempo de duração ainda superior ao planejado anteriormente. De acordo com Baggio e Erdmann (2015) é importante considerar que o cuidar se configura como uma característica inerente ao ser humano, e que para se alcançar qualidade de vida e para se ter condições de cuidar do outro, é necessário primeiramente cuidar de si mesmo. O cuidado processa-se nas interações entre os sujeitos, e envolve suas singularidades, histórias de vida e pluralidades, abrangendo relações que integram pacientes, familiares e diferentes profissionais.

\subsection{Segundo etapa: Dinâmica "Colagem de depoimentos e notícias"}

Esse momento correspondeu a abordagem inicial do conteúdo, com o objetivo de contextualizar o tema a ser trabalhado, e como forma de promover uma reflexão e debate inicial sobre a temática. Para a realização desta etapa, foi mostrado para os participantes o 
depoimento de celebridades nacionais e internacionais que atualmente possuíam transtornos alimentares ou que os haviam apresentado em algum momento da vida e da carreira profissional. Em seguida, foi ressaltado o quanto as redes sociais podem influenciar na concepção que cada pessoa possui com relação ao ideal de beleza, influenciando na insatisfação corporal e contribuindo para a ocorrência destes transtornos. Vale destacar que essa etapa da atividade teve também como propósito demostrar que as figuras públicas, muitas vezes consideradas influências digitais, também possuem problemas físicos e psicológicos que necessitam de tratamento e acompanhamento adequados.

As mídias sociais possibilitam ao usuário não somente gerar o seu próprio conteúdo como também opinar e realizar interferências sobre os demais, tal exposição aumenta significativamente o sentimento de insatisfação e humor negativo, de forma que tempos diários consideráveis dedicados às redes sociais estão associados a maiores comparações e sofrimentos psicológicos, incluindo o desenvolvimento de transtornos de ansiedade e depressão (Brown \& Tiggemann, 2016).

Em grande parte das redes sociais, a avaliação dos indivíduos ocorre por meio da quantidade de curtidas que as postagens e conteúdos recebem. Isso contribui para que os sujeitos precisem investir na aparência recorrendo, muitas vezes, a meios artificiais ou perigosos para transmitir uma determinada imagem, que pode não condizer com a realidade (Baker; Ferszt \& Breines, 2019). Nesse contexto, o debate sobre a importância de utilizar a tecnologia filtrando seus pontos positivos e negativos, aliada a desconstrução de possíveis imposições e ideais físicos de corpo e beleza são essenciais na promoção de uma vida saudável no aspecto social, físico e psicológico, de modo a também garantir o uso seguro da mídia como forma de compartilhar conhecimentos e experiências.

\subsection{Terceira etapa: Exposição do conteúdo dialogado}

Essa etapa consistiu em expor e refletir sobre os assuntos da ação, abordando, principalmente, a definição de Distúrbios da Autoimagem, sinais e sintomas relacionados a esses transtornos, como estes podem contribuir para o desenvolvimento de Transtornos alimentares, diferenças entre bulimia nervosa e anorexia e as possíveis consequências de tais problemas na adolescência e na vida adulta. Vale também ressaltar que a exposição dos assuntos ocorreu de forma dialogada, de modo que os facilitadores tentaram construir um espaço de fala para que os participantes se sentissem à vontade para expressar suas experiências e dúvidas sobre a temática. 
Inicialmente, os organizadores apresentaram a definição de Distúrbios da Autoimagem. Nesse sentido, a American Psychiatric Association (2014), caracterizou o Transtorno Dismórfico Corporal como a percepção alterada e preocupação em falhas na aparência física. De modo que são observados comportamentos repetitivos, entre eles se destacam a constante verificação do próprio corpo no espelho, a busca por tranquilização no uso exagerado de cosméticos, cuidados excessivos com os cabelos, uso de roupas às vezes desconfortáveis, mas que escondam o corpo, além de atos psicológicos em resposta às preocupações com a fisionomia, como a comparação da própria aparência com a de outra pessoa, podendo ocasionar um sofrimento significativo, impactando o indivíduo em áreas sociais, ocupacionais e em outros contextos da vida cotidiana.

Segundo Figueiredo (2019), a construção e reconstrução da imagem corporal é um processo de identificação e formação do adolescente, que perpassa o modo como este se vê e como se relaciona com o seu exterior, não sendo, portanto, exclusiva desta fase, embora possa se tornar significativa nas demais fases. Portanto, a construção da imagem corporal, bem como o entendimento do seu conceito se dará de forma menos conflituosa quando houver maior aceitação e apropriação dos modelos de autoidentificação pelos jovens, pois são nesses momentos que haverá maior busca por figuras idealizadas que se contrapõem aos modelos que se vê da realidade.

Dessa forma, torna-se imprescindível o papel dos pais, profissionais da educação e da saúde em se trabalhar e conversar sobre a imagem corporal inserindo os adolescentes como público alvo, mantendo maior atenção para essa problemática. A escola e a família são extremamente importantes para abordar sobre a identidade corporal e sobre as inadequações dos padrões de beleza em contraposição em se ter uma vida saudável, além de poder expor os riscos que podem acometer a saúde. Recomenda-se que haja a promoção de práticas de educação em saúde nas escolas que envolvam os estudantes e seus responsáveis, para que ocorra o vínculo entre a família, escola e serviços de saúde resultando na minimização dos agravos que impactam os jovens (Sousa; Araújo \& Nascimento, 2016).

Ao longo da discussão sobre a temática houve a inserção e interligação do tema distorção da imagem corporal e transtornos alimentares, de modo que também foram conceituados os transtornos com maior índice de ocorrência, como a bulimia nervosa e anorexia. Ao serem indagados sobre o conhecimento do conceito de tais distúrbios, muitos alunos relataram não saber sobre o assunto ou possuíam um conceito divergente do apresentado na literatura, no entanto se mostraram participativos e bastante interessados sobre a temática. Cabe ressaltar, ainda, que a dificuldade em caracterizar esses tipos de transtornos 
alimentares, por parte dos alunos, ocorreu devido à ausência do debate sobre os mesmos no ambiente escolar e em outros meios de disseminação de informações, como a mídia e o setor familiar.

Ao longo da realização da atividade foi possível estabelecer um momento e espaço de confiança, onde os alunos participantes da atividade se sentiram confortáveis para compartilhar suas experiências pessoais relacionadas a temática abordada, contribuindo para o compartilhamento de vivências e proporcionando uma construção dialogada do conhecimento. Sobre esse aspecto, foi possível perceber os momentos em que parte dos alunos sentiram necessidade de falar sobre a ocorrência de alguns desses transtornos em determinada fase da vida, discernindo os sinais e sintomas que foram apresentados, e relatando, dessa forma, o impacto que tais problemas poderiam causar à saúde.

Diante do exposto, ainda há déficits em se abordar sobre a temática em ambiente escolar e em outros meios em que o adolescente está inserido, infelizmente ainda há escassez, em nossa realidade, em se adotar medidas de suporte que possibilitem a interação entre os jovens e seus familiares, bem como a formação de grupos que possibilitem o debate sobre o assunto. Dessa forma, são perpetuadas falhas de intervenções, impedindo a criação de espaços de fala para o público mencionado, além de fazer com que estes se reclusem em suas condições, dificultando a compreensão e identificação no que diz respeito a esses transtornos na fase vivenciada (Figueiredo, 2019).

Torna-se imprescindível o desenvolvimento de grupos de discussão em ambiente escolar com caráter interdisciplinar, pautando sobre temáticas como a autoaceitação a importância de uma alimentação saudável, bem como ampliar a abordagem desses tipos de transtornos associados pela influência da mídia e criação de redes de apoio em plataformas virtuais, a fim de desmistificar a temática e trabalhá-la com foco no cuidado e atenção do adolescente, com os suportes necessários para a promoção da saúde (Figueiredo, 2019).

Vale ressaltar, o incentivo do debate e de ações educativas voltadas para enfatizar a importância dos exercícios físicos como forma de manutenção da saúde e como ferramenta para manter o corpo saudável, além do incentivo para as mudanças de hábitos de vida, e alerta sobre a exposição dos riscos dos extremos alimentares como a obesidade e a magreza em excesso (Silva; Pontes; Silva \& Silva, 2018).

Por fim, foram observadas algumas dificuldades e limitações para a realização da ação educativa. No que concerne à demanda e as necessidades de cada aluno em consequência do tempo que nos foi proposto, deixando a desejar o aprofundamento do assunto, em alguns 
momentos de debate, além de ser perceptível a necessidade de se abordar a temática com inovações tecnológicas e ações voltadas a necessidade de cada indivíduo.

De acordo com Araújo et al. (2016), há dificuldades de se trabalhar com adolescentes, devido às próprias inconstâncias que detém a fase, havendo maiores anseios de inovação para o desenvolvimento de ações voltadas para estes, seja em ambiente escolar, na Atenção Primária à Saúde e em outros meios que o jovem tenha vínculo para com sua saúde e educação. Por conseguinte, profissionais da saúde aliados com os profissionais da educação, precisam desenvolver ações voltadas para as necessidades e realidade de cada indivíduo, visando buscar facilitar o acesso aos serviços de saúde por meio de programas e serviços voltados para esse público e aprofundamento da temática para além do ambiente escolar.

\subsection{Quarta etapa: Dinâmica final “dúvidas embaixo das carteiras”}

Essa etapa foi elaborada com a finalidade de avaliar se os participantes haviam compreendido a temática e os principais conceitos abordados ao longo da atividade. Dessa forma, foram colocados aleatoriamente, durante a organização do ambiente, papéis abaixo de algumas carteiras onde os alunos iriam estar acomodados. Nos papéis haviam perguntas relacionadas a definição de distúrbios da autoimagem, bem como as definições de anorexia e bulimia, seguidas das características de cada transtorno.

Foi possível perceber que diferentemente do que foi presenciado nos primeiros momentos da atividade, os alunos foram capazes de diferenciar anorexia e bulimia, e puderam destacar com as próprias palavras, como compreendiam os conceitos de autoimagem, e como estes poderiam ser influenciados por diferentes fatores sociais, incluindo os meios de comunicação. De modo que, segundo o relato e as afirmações dos alunos durante esta etapa da atividade, foi possível observar que os participantes compreenderam a importância de filtrar os aspectos positivos e negativos relacionados as tecnologias e os padrões de beleza impostos socialmente.

Dessa forma, como afirma Sabóia et al. (2016), pode-se verificar que o lúdico e dinâmico é capaz de contemplar os critérios para uma aprendizagem considerada efetiva, de modo que desperta a atenção para um determinado assunto e seu respectivo significado, que pode ser posteriormente discutido entre os participantes. Portanto, o conhecimento gerado por meio de uma atividade lúdica caracteriza a transcendência, visto que pode ser transportado para o campo da realidade. 


\section{Considerações Finais}

A atividade representou um enriquecedor momento de aprendizado não somente para os participantes, como também para seus organizadores, visto que foi notória a importância do enfermeiro como educador e agente essencial na promoção da saúde mental de adolescentes e jovens, sendo esse papel ampliado para outros setores e faixas etárias.

A escola que possibilitou a concretização da ação se mostrou disponível e acolhedora para a realização de demais atividades que abordassem e buscassem promover a saúde dos alunos em diferentes contextos. Nesse sentido, destaca-se a necessidade e importância da realização de outras ações educativas e estudos que abordam a temática da saúde mental no contexto escolar, possibilitando um aprofundamento sobre o tema e a realização de intervenções que busquem impactar positivamente na saúde desse público e na prevenção dos casos associados aos distúrbios da autoimagem e suas possíveis consequências.

Também é importante ressaltar que, o papel dos setores educacionais aliados aos de saúde são percebidos como áreas que devem estar articuladas de forma a garantir maior suporte e assistência aos jovens e seus familiares, caracterizando a atuação da equipe multiprofissional no aspecto da atenção psicossocial. A capacitação dos profissionais envolvidos nos serviços e cuidados com relação aos aspectos da autoimagem e dos respectivos transtornos relacionados a esta são uma maneira essencial de garantir uma assistência de qualidade, que considera a saúde sobre seu aspecto multifatorial, como aquela que envolve não somente os contextos físicos, mas que compreende a importância dos aspectos psicológicos e sociais envolvidos.

\section{Referências}

American Psychiatric Association. (2014). DSM-5: Manual diagnóstico e estatístico de transtornos mentais.(5. ed.). Artmed Editora.

Araújo MSD, Sales LKO, Araújo MGD, Morais IFD, Morais FRRD \& Valença CN (2016). Dificuldades enfrentadas por enfermeiros para desenvolver ações direcionadas ao adolescente na atenção primária. Rev. enferm. UFPE on line, 4219-4225. 
Baggio, M. A., \& Erdmann, A. L. (2015). A Circularidade dos Processos de Cuidar e Ser Cuidado na Conformação do Cuidado “do Nós”. Revista de Enfermagem Referência, 7(1), 11 20.

Baker, N., Ferszt, G., \& Breines, J. G. (2019). A qualitative study exploring female college students' Instagram use and body image. Cyberpsychology, Behavior, and Social Networking, 22(4), 277-282.

Bittar, C., \& Soares, A. (2020). Mídia e comportamento alimentar na adolescência. Cadernos Brasileiros de Terapia Ocupacional, 28(1), 291-308.

Brown, Z., \& Tiggemann, M. (2016). Attractive celebrity and peer images on Instagram: Effect on women's mood and body image. Body image, 19(1), 37-43.

Dobrean, A., \& Păsărelu, C. R. (2016). Impact of Social Media on Social Anxiety: A Systematic. New developments in anxiety disorders, 109-129.

Carvalho, A. D. M., Pereira, É. A. A., \& Barros, F. P. (2018). Fatores de risco para síndrome metabólica em adolescentes do ensino médio de uma escola pública do Sul de Minas. Revista da Associação Brasileira de Nutrição.

Carvalho, L. C. D. (2019). A Influência das mídias sociais na imagem corporal e no desenvolvimento de transtornos alimentares em estudantes do sexo feminino da Faculdade Pernambucana de Saúde do Recife-Pe. (Trabalho de Conclusão de Curso). Faculdade Pernambucana de Saúde, Brasil.

Figueiredo, R. (2019). A obsessão pelo corpo e o desenvolvimento de transtornos alimentares em jovens adolescentes. Pretextos-Revista da Graduação em Psicologia da PUC Minas, 4(7), 250-268.

Fontenele, R. M., Ramos, A. S. M. B., Goiabeira, C. R. F., Cutrim, D. S., Galvão, A. P. F. C., \& Noronha, F. M. F. (2019). Impacto dos transtornos alimentares na adolescência: uma revisão integrativa sobre a anorexia nervosa. Revista Enfermagem Atual InDerme, 87(25), 19. 
Abreu Gonçalves, J., Moreira, E. A. M., Trindade, E. B. S. D. M., \& Fiates, G. M. R. (2013). Transtornos alimentares na infância e na adolescência. Revista paulista de pediatria, 31(1), 96-103.

Grealish, A., Tai, S., Hunter, A., Emsley, R., Murrells, T., \& Morrison, A. P. (2017). Does empowerment mediate the effects of psychological factors on mental health, well-being, and recovery in young people?. Psychology and Psychotherapy: Theory, Research and Practice, 90(3), 314-335.

Lima, N. L., Rosa, C. D. O. B., \& Rosa, J. F. V. (2012). Identificação de fatores de predisposição aos transtornos alimentares: anorexia e bulimia em adolescentes de Belo Horizonte, Minas Gerais. Estudos e pesquisas em psicologia, 12(2), 360-378.

Murari, K. S., \& Dorneles, P. P. (2018). Uma revisão acerca do padrão de autoimagem em adolescentes. Revista Perspectiva: Ciência e Saúde, 3(1), 155-168.

Pereira AS et al. (2018). Metodologia da pesquisa científica. [e-book]. Santa Maria. Ed. UAB/NTE/UFSM. Disponível em:

https://repositorio.ufsm.br/bitstream/handle/1/15824/Lic_Computacao_MetodologiaPesquisa-Cientifica.pdf?sequence $=1$.

Ribeiro, K., Silva, J., Santos, M. S., Albuquerque, J. R., \& Pichelli, A. (2015). Vulnerabilidade aos Transtornos Alimentares em Adolescentes: fatores que afetam à satisfação com o corpo. CIAIQ2015, 1(1),328-332.

Sabóia, V. M., Moniz, M. D. A., Daher, D. V., Rangel, E. T., Moura, J. M. B. D., \& Sá, F. C. D. (2016). Dinâmica comunicativa: avaliação da tecnologia educacional sobre drogas com estudantes universitários de enfermagem. Rev Enferm., 24(1), 1-7.

Santos, T. M. (2017). O trabalho do psicólogo no Cras: diferentes formas de cuidar. Revista Pesquisas e Práticas Psicossociais, 13(1), 1-11. 
Silva, I. S. D. S., Pontes, E. D. S.,Silva, M. C. C., \& Silva, E. C. A. (2018). Adolescente e Imagem Corporal: uma Revisão. International Journal of Nutrology, 11(1).

Sousa, A. R., Araújo, J. L., \& Nascimento, E. G. C. (2016). Imagem corporal e percepçao dos adolescentes. Adolescencia e Saude, 13(4), 104-117.

Teixeira, L. A., Freitas, R. J. M. D., Moura, N. A. D., \& Monteiro, A. R. M. (2020). Necessidades de saúde mental de adolescentes e os cuidados de enfermagem: revisão integrativa. Texto \& Contexto-Enfermagem, 29(1), 1-15.

\title{
Porcentagem de contribuição de cada autor no manuscrito
}

\author{
Alicyregina Simião Silva - 16,66\% \\ Janiel Ferreira Felício - 16,66\% \\ Inara da Silva de Moura - 16,66\% \\ Luzia Camila Coêlho Ferreira - 16,66\% \\ José Carlos Gomes de Sousa - 16,66\%
}

Carolina Maria de Lima Carvalho - 16,6\% 